\title{
Effective grain-size of mastery-style online homework levels
}

\author{
Brianne Gutmann, ${ }^{1}$ Noah Schroeder, ${ }^{2}$ Tim Stelzer ${ }^{1}$ \\ ${ }^{1}$ Physics Department, University of Illinois at Urbana-Champaign, 1110 W Green St, Urbana, IL USA \\ ${ }^{2}$ eLearning Design and Services, Indiana University, 535 Michigan St, Indianapolis, IN USA
}

\begin{abstract}
Mastery-style online homework is used in a preparatory physics course at the University of Illinois in Champaign-Urbana. Managing student frustration and making homework content achievable is a priority. In a level that historically has been difficult for students to master, content was broken into two smaller mastery levels for half of the students, with the original level given to the other half. Students performed similarly on follow-up assessments and spent similar amounts of time on the homework and assessments, but significantly more students were able to master the content when they were split into two smaller units. Further, students who saw split levels spent significantly less time re-doing problems that they had previously mastered.
\end{abstract}

\section{INTRODUCTION}

A major component of mastery learning is breaking content into manageable pieces with clear competencies for students to master. Popularized by Bloom [1] and Keller [2], mastery learning delivers small content units to students via repeated testing; students who can demonstrate mastery on a targeted assessment move on to the next unit, while those who are unable to master are given intervening correctives before retesting on a similar test. This pattern continues through as many iterations as necessary. Thus, mastery allows students to spend variable amounts of time on specific content, which adapts to their developing ability and understanding. The mastery delivery method has been tested over the last decades with many positive results, showing improvement in student achievement, retention, and attitudes in both high and low-scoring students and on both low and high-level skills [3]. Mastery-style learning has been used in physics for basic skills recently by Mikula and Heckler [4], but there has been little recent study of mastery-style homework for holistic physics content since a short movement in the 1970s. For interested readers, a more thorough history of mastery-style implementation is summarized in the authors' previous work [5].

At the University of Illinois at Urbana-Champaign, mastery-style online homework replaced traditional online homework in a preparatory course in 2014 [5]. The course was created for engineering students who are identified by a diagnostic test as being underprepared for the calculus-based physics sequence, usually due to deficient or inconsistent high school physics preparation. Because the course attempts to help students from a wide variety of experiences with potentially patchy areas of expertise, mastery is well-suited to allow students extra practice in some areas while also allowing them to move quickly through content they already understand.
Since the original implementation, each iteration of the course gives new opportunity to refine and improve the content and delivery of the mastery-style homework to better serve students. In particular, an emphasis on student affect to temper frustration and incentivize productive behavior was a major consideration from the first year to the second [5]. Unfortunately, there are still many homework levels that the majority of students are unable to master; these levels are a natural place to look for the limits of mastery-style learning. Vygostky's concept of the zone of proximal development (ZPD) encourages learning to take place between what students can and cannot do, but in skills that they can only do with help [6]. If students are unable to master, it is possible that the system is either not providing effective help, or that the material is not well-suited for mastery.

In a previous implementation, it was discovered that students who received extra scaffolding in their problem statement of a difficult mastery level outperformed their classmates (who had not seen extra scaffolding) on a more difficult follow-up assessment (with no scaffolding), while still spending less time overall [7]. Especially because student frustration has historically been of special concern in the course, ensuring that the content is achievable is important.

A common complaint from students is mastery's condition to redo an entire set while only missing one question in the set. Repetition is inherent in mastery, but if the scope of a level is too broad, covering disparate topics, frustration may be warranted. There is also concern that creating levels that are very narrow may make problem solving too disjointed, and students may not see the parts of a problem as being a whole process. This study intends to examine the effects of different grain-size content levels of mastery-style homework on students' behavior and learning. 


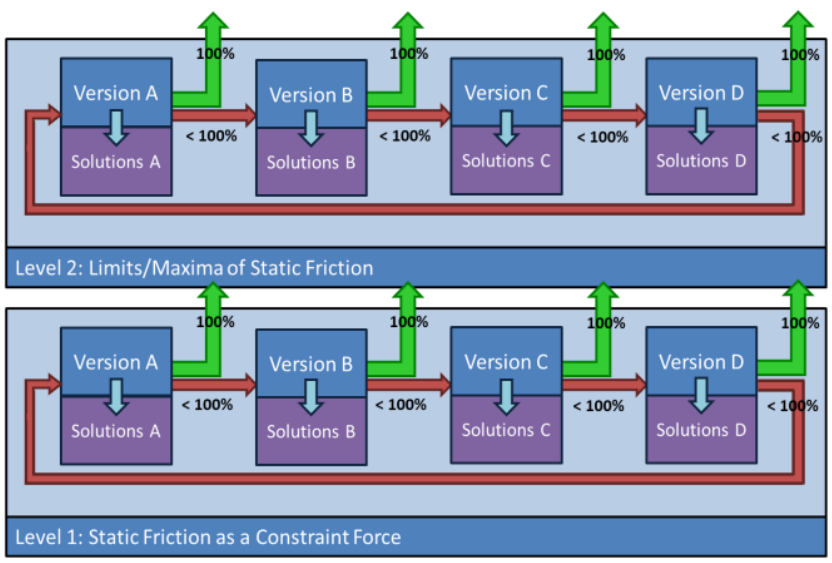

FIG 1. Schematic of online mastery-style homework delivery structure

\section{METHODS}

The experiment was implemented in Physics 100, a preparatory course for students who intend to take the calculus-based physics sequence but are flagged as underprepared. The course is optional and open to anyone but is specifically recommended to students who score below a threshold on a summer physics diagnostic test. Its students are typically first-term freshmen, and enrollment is about 500 students per semester. All students in the course use online mastery-style homework.

A schematic of the delivery method for online masterystyle homework at the University of Illinois is shown in Fig. 1. An assignment consists of several levels, each of which has four versions of several problems intended to test the same competencies but with different surface features. Students are randomly assigned to a first version, which they complete and submit for grading. After submitting the whole

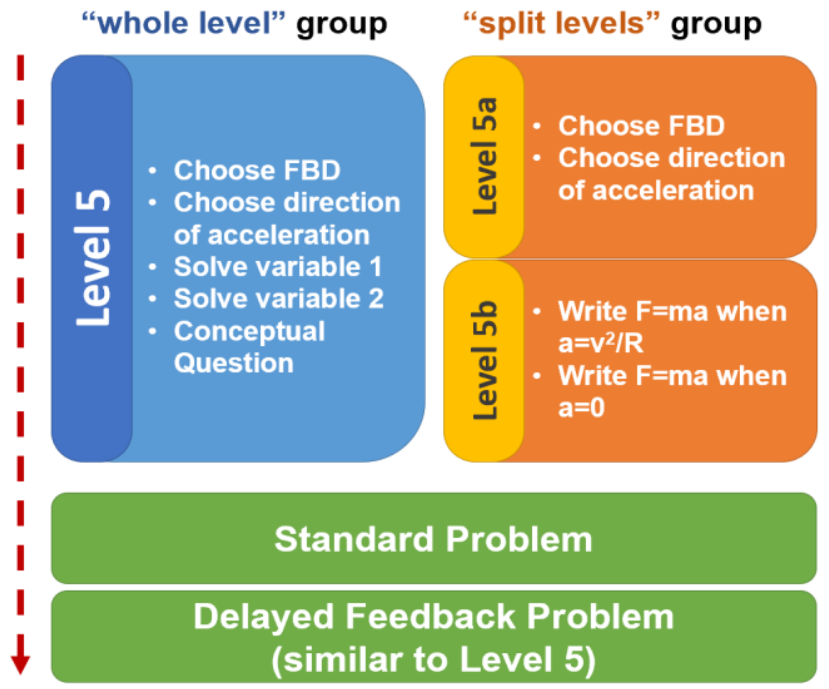

FIG 2. Structure of different treatments and following assessments set, students are told whether they are correct on each problem and narrated animated solution videos become available for all questions (regardless of student correctness on problems). If a student has mastered (answered all questions correctly), they gain access to the next level. If they have not mastered, they are encouraged to study solutions and re-test on a different version. If all four versions are exhausted, students are also moved on and given the best score of their four attempts.

For this study, a difficult level covering uniform circular motion was split into two smaller levels, with half the class receiving the "whole level" (the unaltered difficult level: Level 5) and the other half receiving two "split levels" (Levels 5a \& 5b). Students were divided into groups randomly, with 195 students seeing the "whole level" and 186 students seeing the "split levels." The treatments were the last levels of Week 7, following four levels on friction. Following the different treatments, both groups saw a computational standard problem (immediate feedback with unlimited tries) and a delayed feedback problem (feedback given after the deadline) which was a version of the original "whole level" mastery. Thus, there were only three unique versions of level 5 (since the fourth version was removed to use as the delayed feedback assessment), so only three unique versions of levels $5 \mathrm{a} \& 5 \mathrm{~b}$ were created. The structure of the treatments and assessments for the two groups is shown in Fig. 2; the figure also details how the tasks from the whole level were adapted to smaller levels. Note that the "whole level" group saw conceptual and computational problems that the "split levels" group did not.

\section{RESULTS}

Comparing only the identical assessments (the standard problem and delayed feedback problem), students' performance was statistically equivalent for the two groups.

TABLE 1. Average scores for students with each treatment on common assessments, and total time spent on homework levels, standard problem, and delayed feedback. All are not significant with $\mathrm{p}>0.1$.

\begin{tabular}{ccc}
\hline \hline & $\begin{array}{c}\text { "whole level" } \\
\text { group }\end{array}$ & $\begin{array}{c}\text { "split level" } \\
\text { group }\end{array}$ \\
\hline $\begin{array}{c}\text { Standard Problem } \\
\text { Average Score }\end{array}$ & $80 \pm 2.9 \%$ & $85 \pm 2.7 \%$ \\
$\begin{array}{c}\text { Delayed Feedback } \\
\text { Average Score }\end{array}$ & $56.9 \pm 3.9 \%$ & $56.6 \pm 3.9 \%$ \\
$\begin{array}{c}\text { Average Total } \\
\text { Time Spent }\end{array}$ & $45.9 \pm 2.6 \mathrm{~min}$ & $42.6 \pm 2.1 \mathrm{~min}$ \\
\end{tabular}




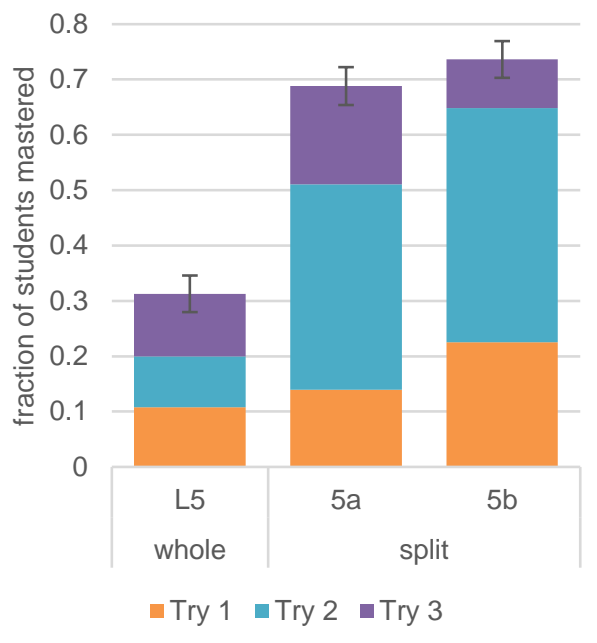

FIG 3. Mastery rate (fraction of students mastering) for whole and split level treatments.

Additionally, the average total time spent by a student in either group on the homework levels, standard exercise, and delayed feedback were also statistically equivalent. Actual values for performance and overall time are shown in Table 1. The distribution of the total time across activities was also similar between the groups.

Within the homework levels themselves, students progressed through the smaller split levels quicker, with a higher rate of mastery overall (around $70 \%$ compared to $30 \%$ ). Students' progression is shown in Fig. 3. The mastery rate for level 5 is significantly different than level $5 \mathrm{a}$ and level $5 \mathrm{~b}$, with effect sizes of 0.8 and 1.0 , respectively, and $\mathrm{p}<0.0001$. To more fairly compare similar content, however, Fig. 4 shows students' progressions through questions 1 and

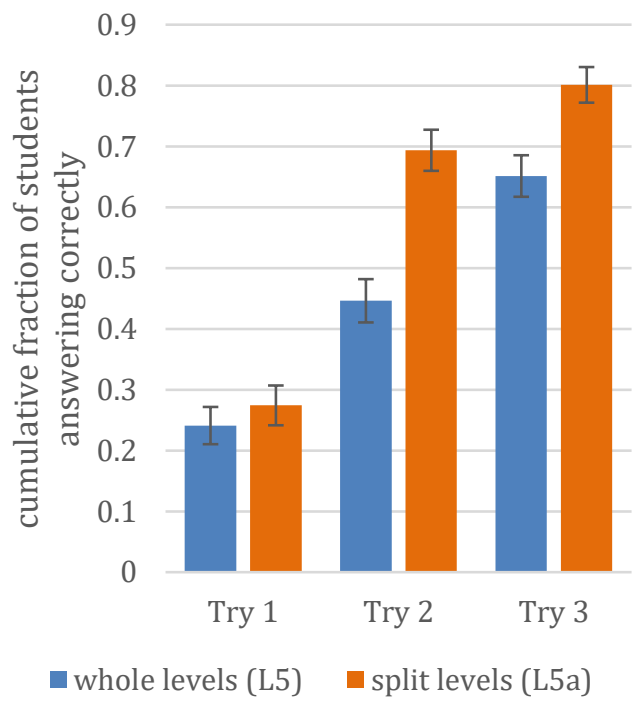

FIG 4. Student progression through similar problems

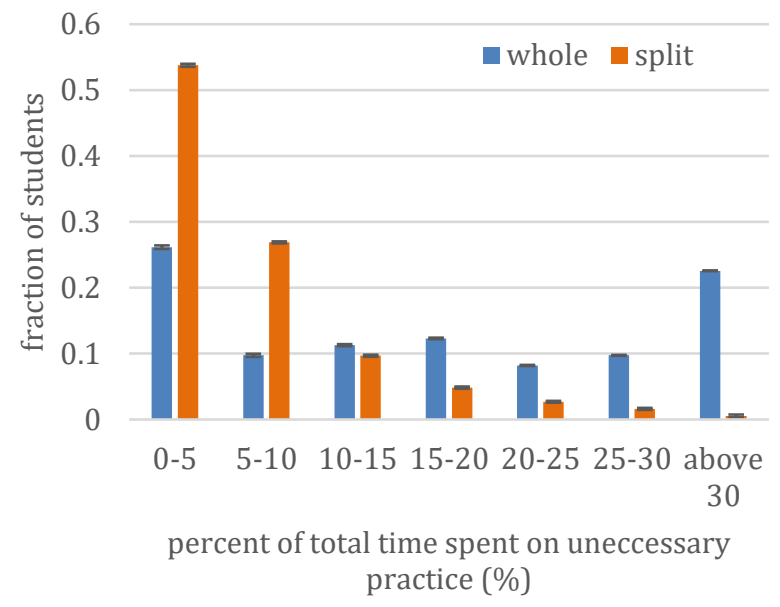

FIG 5. Histogram of percent time students spent on unnecessary practice out of total time spent. Unnecessary practice is defined as time spent re-doing problems they had mastered on a previous version.

2, which were identical question types in level 5 and level 5 a. The group who practiced on the split levels had a higher rate of mastery on their second try by $24.7 \pm 7.0 \%$, corresponding to an effect size of 0.55 and $p<0.0001$. By students' third try, the split levels group had a $15.0 \pm 6.3 \%$ higher rate of mastery on those two problems than their counterparts, corresponding to an effect size of 0.35 with $p<0.005$.

Although students spent similar amounts of time on the homework levels, it was possible to further classify the time they spent as necessary or unnecessary practice. Specifically, unnecessary practice was defined to be time that students spent working on problems that they had mastered on a previous version. By taking a fraction of unnecessary time over the total time students spent on their homework levels, one can see how effectively students used their time with the different treatments. The distribution of students' percentages of unnecessary practice time is shown in a histogram in Fig. 5. Both the average and median of students' unnecessary practice time are more than tripled for the students who saw the whole level compared to the split levels, and the maximum of the distribution for the students seeing the "whole level" reached about 40 minutes of unnecessary practice compared to 20 minutes for "split levels" students. The students who saw the "split levels" averaged $6.0 \pm 0.5 \%$ of their time redoing previously mastered problems, compared to $18.7 \pm 1.2 \%$ for students who worked through the "whole level" mastery. This is an effect size of 1.0 with $\mathrm{p}<0.0001$.

\section{DISCUSSION}

As mastery-style online homework continues to be refined at the University of Illinois, these results encourage 
further study into how changing the grain-size of levels will affect students' performance and frustration. Although there was no evidence that splitting a larger level into smaller pieces improved students' ability to solve problems, we also did not see that it significantly hurt their ability to do multiple-step computational problems. Smaller grain-size levels did, however, allow students to more often master their homework levels in fewer tries, which should help temper student frustration and bolster confidence. Additionally, students were able to prioritize their time more effectively to work on problems that they had not mastered, wasting less time re-doing problems that they can already correctly answer.

These results are good preliminary evidence to probe the research question further for a more definitive result. In its next implementation in Fall 2018, the levels will be redone to be more similar in content. Particularly, the "whole level" group had the benefit of practicing computation and the conceptual questions, while the "split levels" group did not. These skills were tested on the assessments, which may have given the "whole level" group an advantage. In the next experiment, a third level will be added to the split levels which will ask students to practice computation in the context of circular motion, and the conceptual question will be removed from the single level and delayed feedback assessment. The assessments in general will be re-evaluated to include more fine-grained questions which can illuminate where students make mistakes, and how those mistakes correlate to their performance on the homework. Students can also be asked about their level of frustration; it is expected that more successfully mastering levels will make students feel more positively about the homework, but survey questions can give more direct information that was not gathered in the first iteration.

The study will also be duplicated in a static friction level in the same course. The level is a good candidate for this treatment because it is historically difficult and the questions themselves have very different success rates. The variation in question performance suggests that the questions require different skills and that students have different levels of comfort with those different skills.

\section{CONCLUSION}

Moving forward with mastery-style online homework, it is imperative to students' morale for the content to be manageable, particularly for a group that is already at-risk to question their place in engineering. The current focus to improve our mastery-style homework is to mitigate very difficult levels; breaking content into smaller pieces may give students more opportunities to feel success and manage frustration. If doing so does not hurt overall performance, smaller grain-size levels may be a positive solution. Further study with more intentional assessments and an additional area to test the grain-size of the mastery delivery will provide more information about how to split content effectively to help students master content and feel success.

\section{ACKNOWLEDGEMENTS}

This work was partially supported by the NSF through the IUSE program, Grant No. DUE 16-08002.
[1] B. Bloom, Learning for mastery, Eval. Comment 1, 1 (1968).

[2] F. S. Keller, Good-bye teacher..., J. Appl. Behav. Anal. 1, 79 (1968).

[3] C.-L. C. Kulik, J. A. Kulik, and R. L. Bangert-Drowns, Effectiveness of mastery learning programs: A metaanalysis, Rev. Educ. Res. 60, 265 (1990).

[4] B. D. Mikula and A. F. Heckler, Framework and implementation for improving physics essential skills via computerbased practice: Vector math, Phys. Rev. Phys. Educ. Res. 13, 010122 (2017).
[5] B. Gutmann, G. Gladding, M. Lundsgaard, and T. Stelzer, Mastery-style homework exercises in introductory physics courses: Implementation matters, Phys. Rev. Phys. Educ. Res. 14, 010128 (2018).

[6] L. S. Vygotsky, Mind and Society: The Development of Higher Mental Processes (Harvard University Press, Cambridge, MA, 1978).

[7] B. Gutmann, G. Gladding, M. Lundsgaard, and T. Stelzer, Mastery learning in the zone of proximal development, 2017 PERC Proceedings [Cincinnati, OH, July 26-27, 2017], edited by L. Ding, A. Traxler, and Y. Cao 